\title{
Organizing Offshoring:
}

\section{Middle Managers and Communication Costs}

\author{
Pol Antràs, Luis Garicano and Esteban Rossi-Hansberg*
}

January 28,2006

\begin{abstract}
Why do firms decide to offshore certain parts of their production process? What qualifies certain countries as particularly attractive locations to offshore? In this paper we address these questions with a theory of international production hierarchies in which teams arise endogenously to make efficient use of agents' knowledge. Our theory highlights the role of host-country management skills (middle management) in bringing about the emergence of international offshoring. By shielding top management in the source country from routine problems faced by host country workers, the presence of middle managers improves the efficiency of the transmission of knowledge across countries. The model further delivers the prediction that the positive effect of middle skills on offshoring is weaker, the more advanced are communication technologies in the host country. We provide evidence consistent with this prediction.
\end{abstract}

${ }^{*}$ Harvard University, University of Chicago, and Princeton University, respectively. We are thankful to Daron Acemoglu for a very helpful discussion at the AEA meetings and for encouraging us to explore the issues studied in this paper. 


\section{INTRODUCTION}

Recent developments in the world economy have led to a staggering disintegration of the production process across borders. ${ }^{1}$ Why do firms decide to offshore certain parts of their production process to foreign locations? What qualifies certain countries as particularly attractive locations to offshore? In this paper we address these questions with a theory of production hierarchies in which cross-country differences in the distribution of skills, as well as differences in the cost of transmitting knowledge internationally versus locally, determine the decision to offshore or not to a particular country.

Our model illustrates how the decision to offshore parts of the production process to foreign countries may be associated with changes in the organizational structure of firms, as firms may introduce intermediate layers of managers to minimize the costs of transmitting knowledge across borders. More specifically, our theory describes situations in which international offshoring to a particular host country is only profitable if the production facility in the host country is composed of two layers rather than one: A set of workers specialized in production and a set of middle managers in charge of supervision. By shielding the top management in the home country from having to deal with routine problems faced by workers in the host country, the presence of middle managers allows a more efficient (time-saving) transmission of knowledge across countries.

As in Antràs, Garicano and Rossi-Hansberg (2006), we develop these ideas in a general equilibrium framework with two countries, the North and the South. We model a world economy in which production requires time and knowledge, and where agents with heterogenous abilities sort into teams competitively. The distribution of skills in the host country (the South) plays a central role in the analysis. In particular, in situations in which the efficient organization of production demands the presence of middle managers in the host country, the availability of "middle skills" in the host country becomes crucial for attracting offshoring.

We show however that the availability of "middle skills" is not always conducive to offshoring into a particular country. When the communication technologies available to southern teams are sufficiently developed, the presence of middle skills may actually hinder the emergence of offshoring. The intuition for this result is that advanced southern communication technologies foster the formation of domestic teams in the host country, thus increasing the opportunity cost and equilibrium remuneration of local agents hired by multinational firms. ${ }^{2}$ We show that this increase in the opportunity cost of host-country

\footnotetext{
${ }^{1}$ See Feenstra (1998) or the more recent but less formal account in Friedman (2005).

${ }^{2}$ In the model, whether host-country domestic teams are formed or not depends on the level of southern communication costs. We stick to this interpretation in the empirical section of the paper, but it should be clear that, in the real world, other technological and institutional factors play an important role in fostering or hindering the formation of such teams.
} 
agents may be large enough to altogether deter offshoring to that particular country.

Our analysis thus shows that the distribution of skills in host countries, together with local production possibilities, are crucial in determining the desirability of a country as a target of offshoring. We examine the empirical validity of one of the main predictions of the model using data on average FDI inflows and educational attainment measures in a large cross-section of countries in the period 1993-2002. After constructing an index of the availability of communication technologies in different countries, we show that consistently with the model, a higher availability of middle skills (as measured by secondary school enrollment) is associated with higher FDI inflows (as a percentage of GDP) into countries with poor communication technologies, but with lower FDI inflows into countries with advanced communication technologies. ${ }^{3}$

This paper is most closely related to our previous work in Antràs, Garicano and RossiHansberg (2006), where we developed the notion of international offshoring as being the outcome of the assignment of heterogeneous agents into international hierarchical teams. In that paper we focused however on an analysis of the consequences of international team formation for the matching between managers and workers and for the implied structure of wages. For that purpose, we simplified our analysis by focusing on two-layer teams and by drawing no distinction between international and local communication costs within multinational and in southern teams. As a consequence of these features, the model ruled out any active role of host-country middle managers and always generated international offshoring in equilibrium, being thus unable to shed light on the extensive margin of offshoring. 4

The remaining of the paper contains six sections. In Section II, we describe the general setup. In Section III, we analyze the emergence of offshoring in a model where a host country (the South) has very limited opportunities of production and we illustrate the positive role of middle managers in bringing about offshoring. In Section IV, we look at the other polar case in which the North and the South share access to the same communication technologies, while in Section $\mathrm{V}$ we consider intermediate cases. Section

\footnotetext{
${ }^{3}$ Our results also seem to accord well with casual discussions of particular offshoring decisions. For instance, Spar (1998) and Larraín, López-Calva, and Rodríguez-Clare (2001) describe the decision of Intel to locate a microprocessor plant in Costa Rica in 1996. Intel was considering four alternative locations in Latin America: Argentina, Brazil, Chile, and Mexico. Both these studies describe the main factors that made Intel finally decide to locate the plant in Costa Rica despite frequent bottlenecks in telecommunication and electrictricity services in the country. The authors emphasize the availability of highly-trained graduates in Costa Rica as being one of the decisive factors. Further evidence of the importance that Intel gave to "middle skills" comes from the active involvement of the company in redefining the curricula of the country's technical high schools and advanced training programs (Spier, 1998, p. 19)

${ }^{4}$ Our model is also related to models of vertical FDI and outsourcing, where the incentive to fragment the production process is driven by factor-price differentials (see Helpman, 1984, Yeaple, 2003, and Antràs, 2003).
} 
VI summarizes the main empirical implication of the theory and contrasts it with the data. The last section concludes.

\section{GENERAL SET UP}

The model builds on Antràs, Garicano and Rossi-Hansberg (2006) -AGR hereafter-, which in turn builds on Garicano and Rossi-Hansberg (2005). The framework here is simpler in that we assume a discrete number of skill levels (rather than a continuum). The model is however more general than AGR in that (i) we allow for the formation of teams with more than two layers (which is a prerequisite for studying the role of middle management); and (ii) we introduce differences between the costs of transmitting knowledge locally and internationally.

The world economy is inhabited by a unit measure of agents, each endowed with a skill level $z \in[0,1]$ and a unit of time. The distribution of skills in the world population is given by the distribution function:

$$
s(z)=\left\{\begin{array}{lll}
s_{h} & \text { if } & z=z_{h} \\
s_{m} & \text { if } & z=z_{m} \\
s_{l} & \text { if } & z=z_{l}
\end{array}\right.
$$

with $z_{h}>z_{m}>z_{l}$ and $s_{h}+s_{m}+s_{l}=1 .^{5}$

The world consists of two countries: the North and the South. As in AGR, we assume that the North and the South are endowed with different distributions of skills, with the North being endowed with relatively higher skill levels. We capture this feature in a stark way: all agents in the North are endowed with a skill level equal to $z_{h}$, while agents in the South are endowed with a skill level equal to $z_{m}$ or $z_{l}{ }^{6}$ Our assumptions on the distribution of skills lead to a stylized model of the decision of high skilled northern agents on whether to offshore to the South or remain self-employed. ${ }^{7}$

Agents derive linear utility from consuming the only good in the economy, whose price is normalized to one. Production of this good combines labor and knowledge. As in Garicano (2000) and Garicano and Rossi-Hansberg (2005), production requires solving the problems that arise in production. An agent with skill $z$ can solve all problems indexed between 0 and $z$, so an agent with skill $z^{\prime}>z$ can solve all the problems that $z$ can solve plus some extra ones. That is, knowledge is cumulative. We normalize the

\footnotetext{
${ }^{5}$ Garicano and Rossi-Hansberg (2006) use a similar framework, with a discrete number of skill levels, to study the emergence of large US corporations at the turn of the 20th century.

${ }^{6}$ This implies that the relative size of the North is given by $s_{h} /\left(s_{m}+s_{l}\right)$.

${ }^{7}$ It would be straightforward to extend the analysis and allow domestic team formation in the North. This would however substantially increase the taxonomy of cases to consider without providing many insights into the role of southern skills to foster offshoring.
} 
set of problems so that the skill level $z$ is also the proportion of problems an agent can solve. Agents have one unit of time that they can use in production or communicating with other agents. Agents face one problem per unit of production time and we normalize output so that a solved problem yields one unit of output.

Agents can choose to produce together in teams or work on their own (self-employment). A self-employed agent with knowledge $z$ spends all his time in production and solves a fraction $z$ of the problems that he confronts. Hence, his expected output and income is given by $y=z$. Agents producing in teams can communicate their knowledge to others, and thus help them solve problems. This possibility allows them to form organizations in which several individuals combine their time and knowledge to produce together. Such organizations are composed by production workers, who draw problems, and problem solvers (managers), who can answer questions and thus help workers solve the problems they cannot solve on their own. Agents are income maximizers and so choose the occupation that pays them the highest wage given their ability.

Workers draw a problem and try to solve it. If they can, they produce; if they cannot, they ask for help to the managers right above them, in which case these managers incur a communication cost $h_{i} \in(0,1)$, where $i$ denotes the identity and location of the parties communicating (which we will specify below). If the manager knows the solution to the problem, the team produces one unit of output. If the manager does not know the solution but has a manager above him, he asks this manager for help, and this upper-level manager incurs a communication cost $h_{j}$ (more on this below). In such a case, production occur only if the upper-level manager knows the solution to the problem. The skill distribution we assume above, with only three levels of skill, implies that three organizational forms can potentially arise in equilibrium, namely, three-layer teams, two-layer teams, and selfemployed agents. ${ }^{8}$ Hence, the above discussion suffices to cover the workings of all possible production teams.

To summarize, production is organized in knowledge-based hierarchies, with some agents specialized in production and some in management. This production structure also gives rise to "management by exception," whereby production workers deal with the most common problems and problem solvers with the exceptions. These characteristics are optimal under the assumption that agents do not know who may know the solution to problems they cannot solve, as Garicano (2000) shows in a model with homogeneous workers. The purpose of the hierarchy is to protect the knowledge of those who are more knowledgeable from easy questions others can solve.

Communication costs depend on the circumstance in which communication occurs. We

\footnotetext{
${ }^{8}$ More specifically, it is never optimal to assign two agents with the same skill level to different layers of an organization (or to have subordinates with higher talent than managers). The reason is that then managers would not increase the output of subordinates but the cost of production would increase by their wage (see Garicano and Rossi-Hansberg, 2005).
} 
denote communication costs within southern teams that are not part of a multinationals by $h_{S}$. Communication costs between southern agents within a multinational are denoted by $h_{L}$, and international communication costs by $h_{I}$. We assume throughout that international communication costs are higher than local communication costs within multinational teams, and so $h_{I}>h_{L}$. We also assume that local communication technology within multinational teams is at least as good as southern communication technology available to agents belonging to southern teams, and so $h_{L} \leq h_{S}$. Hence, multinationals will provide two different inputs to agents in the South: first, the ability to benefit from the knowledge of high-skilled agents in the North; and second, access to a (weakly) better technology for local communication. We interpret the latter feature as a form of technology transfer from multinationals to the South. ${ }^{9}$ As we will see below the relative quality of the local multinational and southern technologies will be crucial in the analysis.

Let us illustrate how production in a two-layer team is carried out. A top manager with knowledge $z_{2}$ can lead a team of $n_{0}$ production workers. These workers draw one problem each, and solve a fraction $z_{0}$ of them. Hence they pass on a fraction $\left(1-z_{0}\right)$ of all problems. Managers are thus asked to solve $n_{0}\left(1-z_{0}\right)$ problems, which they can address in $n_{0}\left(1-z_{0}\right) h_{i}$ units of time. Optimally, managers join teams with precisely the right number of production workers so that they use all their time. Since all agents have one unit of time available, the team size $n_{0}$ is implicitly given by

$$
n_{0} h_{i}\left(1-z_{0}\right)=1
$$

where $i \in\{I, L, S\}$. The time constraint implies that the span of the manager is limited by the knowledge of their subordinates.

Output is produced whenever either workers or manager know the solution to the problems, so

$$
y=z_{2} n_{0} .
$$

Note the source of complementarity between skills in our model: An able top manager increases the productivity of all workers in the team. At the same time, the more knowledgeable are subordinates, the larger the team and the more can managers leverage their knowledge.

Denote the earnings of workers by $w_{0}$. Then zero profits implies that the wage of managers is given by

$$
w_{2}=n_{0}\left(z_{2}-w_{0}\right)=\frac{z_{2}-w_{0}}{h_{i}\left(1-z_{0}\right)} .
$$

Production in three-layer teams is similar but it includes a measure $n_{1}$ of middle managers. Let their skill level be given by $z_{1}$. Then if the skill of workers is given by $z_{0}$, the

\footnotetext{
${ }^{9}$ This feature is also consistent with widely available data suggesting that foreign affiliates of multinational firms appear to be more productive than comparable domestic firms in the same host country (see for instance, Aitken and Harrison, 1999).
} 
top manager is only asked to solve $n_{0}\left(1-z_{1}\right)$ problems, while the layer of middle managers are asked to solve $n_{0}\left(1-z_{0}\right)$ problems. The time constraints of these two-layers of managers are thus

$$
n_{0} h_{i}\left(1-z_{1}\right)=1
$$

and

$$
n_{0} h_{j}\left(1-z_{0}\right)=n_{1}
$$

where $h_{j}$ for $j \in\{I, L, S\}$ denotes the cost of communicating knowledge from top to middle managers. These two equations pin down the size of each of the two lower layers in the team.

Denoting the earnings of middle managers by $w_{1}$, we have that

$$
w_{2}=n_{0}\left(z_{2}-w_{0}\right)-n_{1} w_{1}=\frac{z_{2}-w_{0}-h_{j}\left(1-z_{0}\right) w_{1}}{h_{i}\left(1-z_{1}\right)} .
$$

In the next three sections, we turn to analyze the equilibrium of our two-country world economy, where all agents maximize utility and labor markets clear in both countries. We denote by $w_{i}$ the earnings of an agent with skill $z_{i}$. Note that if the equilibrium is such that a fraction of agents with skill level $z_{i}$ remain self-employed, then the equilibrium wage of all agents with that skill level necessarily equals $w_{i}=z_{i}$.

As mentioned above, the simple skill distribution we have assumed implies that we can focus on studying three-layer teams, two-layer teams, and self-employment. This is because it is never optimal to assign two agents with the same skill level to different layers of an organization. Similarly, a team will never have a manager that is less skilled than his subordinates. In terms of the specifics of our two-country model, this implies that northern agents with skill $z_{h}$ will either be self-employed or top managers, while southern agents with skill $z_{l}$ will either be self-employed or workers. Southern agents with skill $z_{m}$ may be top managers, middle managers, workers or self-employed.

We shall assume throughout that $s_{h}$ is sufficiently low relative to $s_{m}$ and $s_{l}$, which ensures that high-skilled northern agents are self-employed only in situations in which all other agents in the world economy are also self-employed.

\section{EQUILIBRIUM WITH INEFFICIENT SOUTHERN COMMUNICATION}

We start by analyzing a situation in which the local communication costs $h_{S}$ faced by southern agents in domestic teams are so high that domestic southern teams never form. Namely, $h_{S}$ is such that

$$
\frac{z_{m}}{h_{S}\left(1-z_{l}\right)}<z_{m}+\frac{z_{l}}{h_{S}\left(1-z_{l}\right)}
$$


or

$$
h_{S} \geq \widehat{h}_{S} \equiv \frac{z_{m}-z_{l}}{z_{m}\left(1-z_{l}\right)} .
$$

In words, total production in a local southern team is smaller than what its members can get if they work as self-employed. This leads to a world economy that will be in one of four possible equilibria: ${ }^{10}$

\section{No Offshoring}

This corresponds to a situation in which agents in the North do not find profitable to form two or three-layer teams with agents in the South. In such a case, all agents are necessarily self-employed (since middle- and low-skilled agents do not form teams in the South). This implies that all agents earn their self-employment wages:

$$
w_{i}=z_{i} \text { for } i=h, m, l \text {. }
$$

\section{Two-Layer Middle Skill Offshoring $\left(z_{0}=z_{m}\right)$}

In this case, northern agents decide to form international teams, but only with southern middle-skilled agents (who become workers). Northern agents thus earn a wage equal to

$$
w_{h}=\frac{z_{h}-w_{m}}{h_{I}\left(1-z_{m}\right)}
$$

where $w_{m}$ refers to the wage of southern middle-skilled agents. It is clear that agents with skill $z_{l}$ will in this case be self-employed and thus $w_{l}=z_{l}$. Furthermore, notice that equation (1) with $h_{i}=h_{I}$ pins down the relative share of agents of each type in a two-layer team as a function of parameters. It will thus be the case that, for sufficiently low $s_{h}$ (our assumption above), a fraction of medium-skilled agents will also remain unemployed in equilibrium, and thus $w_{m}=z_{m} \cdot{ }^{11}$

\section{Two-Layer Low Skill Offshoring $\left(z_{0}=z_{l}\right)$}

This case is similar to the one above, but now northern agents form teams with lowskilled southern agents. The wages of the northern agents are in this case given by

$$
w_{h}=\frac{z_{h}-w_{l}}{h_{I}\left(1-z_{l}\right)} .
$$

In addition, it is clearly the case that $w_{m}=z_{m}$ (all medium-skilled southern agents are self-employed), and for sufficiently low $s_{h}$, a fraction of the low-skilled southern agents will also be self-employed, implying that $w_{l}=z_{l}$.

\footnotetext{
${ }^{10}$ We ignore the possibility of "mixed equilibria," with some of the four situations below coexisting. The discussion below should make clear that, for a sufficiently low fraction $s_{h}$ of high-skilled agents, these mixed equilibria can only happen in knife-edge cases.

${ }^{11}$ What do we mean by a "sufficiently low $s_{h}$ "? For this particular case, the condition for mediumskilled agents to be in excess supply at a wage higher than $z_{m}$ is given by $s_{h}<s_{m} h_{I}\left(1-z_{m}\right)$. Analogous conditions can be derived for the other cases.
} 


\section{Three-Layer Offshoring}

In this case, agents in the North form three-layer teams and obtain a wage given by

$$
w_{h}=\frac{z_{h}-w_{l}-w_{m} h_{L}\left(1-z_{l}\right)}{h_{I}\left(1-z_{m}\right)} .
$$

Notice from equations (2) and (3) that the relative share of agents of each type in these teams is fixed, in the sense that it is pinned down by parameters. It will thus (generically) be the case that a fraction of agents of at least two types will end up being self-employed in equilibrium, and the wages of these two types will then be determined by their selfemployment wages. For low enough $s_{h}$, it will necessarily be the case that all agents in the South will earn their self-employment wages: $w_{m}=z_{m}$ and $w_{l}=z_{l}$.

\section{Communication Costs, Middle Skills and Offshoring}

Having described these four potential types of equilibria, let us study when they emerge in equilibrium. First note that high-skilled agents in the North always prefer to form twolayer teams with low-skilled agents than two-layer teams with medium-skilled agents. That is,

$$
\frac{z_{h}-z_{l}}{h_{I}\left(1-z_{l}\right)}>\frac{z_{h}-z_{m}}{h_{I}\left(1-z_{m}\right)}
$$

for all $z_{m}>z_{l}$. This implies that given our assumptions on the supply of skills, equilibria of type 2 never arise. ${ }^{12}$

Next note that an equilibrium with three-layer offshoring requires

$$
\frac{z_{h}-z_{l}-z_{m} h_{L}\left(1-z_{l}\right)}{h_{I}\left(1-z_{m}\right)}>\max \left\{z_{h}, \frac{z_{h}-z_{l}}{h_{I}\left(1-z_{l}\right)}\right\} \text {. }
$$

Straightforward differentiation implies that the left-hand side of this inequality is increasing in middle skills $z_{m}$ if and only if

$$
h_{L}<\widehat{h}_{L} \equiv \frac{z_{h}-z_{l}}{1-z_{l}} .
$$

In that case, when $z_{m}$ is close to $z_{l}$, (5) will not hold, while when $z_{m}$ is sufficiently large, (5) will necessarily hold. Hence, there exist a unique threshold skill level $\widehat{z}_{m} \in\left(z_{l}, 1\right)$ over which three-layer offshoring is an equilibrium and under which it is not. The threshold $\widehat{z}_{m}$ is obtained by setting (5) to equality.

In the converse case in which $h_{L}>\widehat{h}_{L}$, one can easily verify that condition (5) cannot possibly hold for any $z_{m} \in\left(z_{l}, 1\right)$, and thus three-layer offshoring cannot be an equilibrium. ${ }^{13}$ In such case, the equilibrium will entail no offshoring or two-layer offshoring. In

\footnotetext{
${ }^{12}$ Note that this is not inconsistent with skill stratification and positive sorting in more general setups like Garicano and Rossi-Hansberg (2005).

${ }^{13}$ This is because, when $h_{L}>\left(z_{h}-z_{l}\right) /\left(1-z_{l}\right)$, the left-hand side of $(5)$ is decreasing in $z_{m}$, and the condition is not satisfied for $z_{m}=z_{l}$.
} 
particular, no offshoring is preferred to two-layer offshoring if international communication costs are high $z_{h}>\left(z_{h}-z_{l}\right) /\left[h_{I}\left(1-z_{l}\right)\right]$, or

$$
z_{h} h_{I}>\widehat{h}_{L}
$$

while two-layer offshoring is preferred to no offshoring when the converse of condition (7) holds. ${ }^{14}$

Formally stated, if we define offshoring as the volume of production in multinational teams, we have shown that:

Proposition 1 If $h_{S} \geq \widehat{h}_{S}$, there exists two thresholds $\widehat{h}_{L} \in(0,1)$ and $\widehat{z}_{m} \in\left(z_{l}, 1\right)$ such that:

(i) Three-layer offshoring is an equilibrium if and only if $h_{L}<\widehat{h}_{L}$ and $z_{m}>\widehat{z}_{m}$.

(ii) Otherwise, offshoring is independent of $z_{m}$. If $z_{h} h_{I}>\widehat{h}_{L}$ there is no offshoring in equilibrium, while if $z_{h} h_{I}<\widehat{h}_{L}$ two-layer offshoring is an equilibrium.

Because the choice between no offshoring and two-layer offshoring is independent of $z_{m}$, we can thus conclude that a larger $z_{m}$ tends to (weakly) favor the emergence of an equilibrium with offshoring. ${ }^{15}$ In addition, the output of offshoring teams is (weakly) increasing in the skill level of middle managers. Therefore, we can conclude that:

Corollary 1 If $h_{S} \geq \widehat{h}_{S}$, in equilibrium offshoring is (weakly) increasing in the skill level $z_{m}$ of middle-skilled agents.

That is, better middle skills improve the ability of middle managers to protect top managers in the North from "expensive" routine problems, thus making offshoring more attractive.

\section{EQUILIBRIUM WITH EFFICIENT SOUTHERN COMMUNICATION}

In the previous section we have shown that the existence of "middle skills" in the South fosters international offshoring. This section briefly illustrates that this result heavily

\footnotetext{
${ }^{14}$ Note that in order for three-layer teams to be necessary for the emergence of offshoring we need that both $z_{m}>\widehat{z}_{m}$ and

$$
z_{h} h_{I}>\widehat{h}_{L}>h_{L}
$$

In words, we need that middle-skilled agents in the South are relatively able and that the cost of communicating knowledge across borders is large relative to the cost of communicating knowledge within borders.

${ }^{15}$ Since $\widehat{h}_{S}$ depends on $z_{m}$, increases in $z_{m}$ may move the equilibrium away from the set in which the proposition applies, namely, $h_{S} \geq \widehat{h}_{S}$. However, we will show below (Proposition 2) that when $h_{S}<\widehat{h}_{S}$ the equilibrium has the same properties.
} 
relies on our assumption that domestic team formation is limited by high local southern communication costs.

To see this, consider the case in which $h_{S}=h_{L}$ and so local communication costs are the same no matter if local communication happens within multinational teams or within southern domestic teams. Relative to the previous section, the only new feature is that an equilibrium may now feature two-layer teams between agents with skill $z_{m}$ and $z_{l}$. The ability to form local teams imposes the following restriction on southern wages,

$$
w_{m} \geq \frac{z_{m}-w_{l}}{h_{L}\left(1-z_{l}\right)} .
$$

If this condition was not satisfied in equilibrium, southern agents would have incentives to deviate from that equilibrium and form two-layer teams among themselves. Using condition (8) it thus follows that the rents that northern agents with skill $z_{h}$ obtain from three-layer offshoring must satisfy

$$
w_{h}=\frac{z_{h}-w_{l}-w_{m} h_{L}\left(1-z_{l}\right)}{h_{I}\left(1-z_{m}\right)} \leq \frac{z_{h}-z_{m}}{h_{I}\left(1-z_{m}\right)}<\frac{z_{h}-z_{l}}{h_{I}\left(1-z_{l}\right)} .
$$

With the possibility of the formation of two-layer southern teams, there is an additional instrument to clear factor markets, and (generically) the equilibrium will now feature only one type of agents being (partially) self-employed. Condition (9) above implies that whenever some agents with skills $z_{m}$ or $z_{l}$ are self-employed (which will be the case whenever $s_{h}$ is low, as we have been assuming throughout), then three-layer offshoring will be dominated by two-layer international offshoring. ${ }^{16}$

The intuition behind this result is that when agents in the South have the option of forming teams between themselves, the opportunity cost of forming three-layer international teams increases to the point where these become unprofitable. ${ }^{17}$

How do medium skills affect the extensive margin of offshoring in this case? It is straightforward to see that whenever low-skilled southern agents are partially self-employed, then $w_{l}=z_{l}$. And since $w_{m} \geq z_{m}$, two-layer offshoring will only emerge if it involves lowskilled workers. The analysis of this case is as in the previous section, with offshoring emerging only if $h_{L}<\widehat{h}_{L}$, which is independent of $z_{m}$. If, alternatively, middle-skilled southern agents are partially self-employed then it is no longer clear which type of twolayer offshoring will emerge in equilibrium. This depends on a relative comparison of $\left(z_{h}-z_{m}\right) /\left[h_{I}\left(1-z_{m}\right)\right]$ and $\left[z_{h}-z_{m}\left(1-h_{L}\left(1-z_{l}\right)\right)\right] /\left[h_{I}\left(1-z_{l}\right)\right]$. Regardless, of the

\footnotetext{
${ }^{16}$ Note also that when high-skilled northern agents are (partially) self-employed, then they will earn the same wage regardless of the equilibrium organization. They are thus indifferent between different organizational modes.

${ }^{17}$ This is not the case in Garicano and Rossi-Hansberg (2005) where the incentives for top managers to acquire knowledge increase with the size of their teams, making three (or more) layer teams profitable even though agents in lower layers can organize by themselves.
} 
form of two-layer offshoring, it is clear however that a larger $z_{m}$ will reduce the attractiveness of offshoring versus no offshoring.

This section has therefore shown that the emergence of offshoring, and in particular that of three-layer offshoring, depends crucially on inefficient local communication technology in the South. If this technology is as good as the one used by multinationals, better medium-skilled agents imply better local teams, not more offshoring! On the contrary, with good local communication the model is actually consistent with better middle skills in the South reducing the attractiveness of offshoring.

\section{THE INTERMEDIATE CASE}

Let us finally consider intermediate cases. In particular, consider the case in which the technology to communicate in the South is less efficient than the local communication technology of multinationals $\left(h_{S}>h_{L}\right)$. The formation of southern teams now imposes the constraint

$$
w_{m} \geq \frac{z_{m}-w_{l}}{h_{S}\left(1-z_{l}\right)} .
$$

When $h_{S}$ is high enough $\left(h_{S} \geq \widehat{h}_{S}\right)$, two-layer southern teams will not be formed in equilibrium and the analysis is as in Section III. For lower $h_{S}\left(h_{S}<\widehat{h}_{S}\right)$, these teams will be formed and will ensure that only one type of agent is self-employed in equilibrium. Let us focus on these situations hereafter.

We next consider the four cases discussed in Section III, but now taking into account that the wages of southern agents will satisfy (10) with equality. To simplify the exposition, we will focus on the case in which $s_{l}$ is high enough to ensure that low-skilled southern agents are partially self-employed, and thus $w_{l}=z_{l}$ and $w_{m}=\left(z_{m}-z_{l}\right) /\left[h_{S}\left(1-z_{l}\right)\right]$. We briefly consider an alternative scenario at the end of this section.

As in Section III, we begin by noting that since $w_{m}>z_{m}$ whenever $h_{S}<\widehat{h}_{S}$, twolayer offshoring with middle-skilled southern agents will again be dominated by two-layer offshoring with low-skilled southern agents. Furthermore, since $w_{l}=z_{l}$, the comparison between this latter option and no offshoring is identical to that discussed in Section III and no offshoring will dominate two-layer offshoring whenever $z_{h} h_{I}>\widehat{h}_{L}$ (where remember that $\left.\widehat{h}_{L} \equiv\left(z_{h}-z_{l}\right) /\left(1-z_{l}\right)\right)$ and vice versa when $z_{h} h_{I}<\widehat{h}_{L}$.

Next note that an equilibrium with three-layer offshoring requires

$$
\frac{z_{h}-z_{m} \frac{h_{L}}{h_{S}}-z_{l}\left(1-\frac{h_{L}}{h_{S}}\right)}{h_{I}\left(1-z_{m}\right)}>\max \left\{z_{h}, \frac{z_{h}-z_{l}}{h_{I}\left(1-z_{l}\right)}\right\} .
$$

Straightforward differentiation implies that the left-hand side of this inequality is increasing in middle skills $z_{m}$ if and only if

$$
h_{L} / h_{S}<\widehat{h}_{L}
$$


This condition is analogous to (6) in Section III, but it also applies to cases in which $h_{S}$ is not prohibitively high (i.e., $h_{S}<1$ ). Moreover, it is again the case that provided that $(12)$ holds, when $z_{m}$ is close to $z_{l}$, (11) will not hold, while when $z_{m}$ is sufficiently large, (11) will necessarily hold. Hence, there again exists a unique threshold skill level $\bar{z}_{m} \in\left(z_{l}, 1\right)-$ obtained by setting (11) to equality - over which three-layer offshoring is an equilibrium and under which it is not.

Following the same logic as in Section III, one can show that in the converse case in which $h_{L} / h_{S}>\widehat{h}_{L}$, condition (11) cannot possibly hold for any $z_{m} \in\left(z_{l}, 1\right)$, and thus three-layer offshoring cannot be an equilibrium. In such case, the equilibrium will entail no offshoring or two-layer offshoring, with the choice determined by the relative size of $z_{h} h_{I}$ and $\widehat{h}_{L}$.

Formally stated we have shown that:

Proposition 2 If $h_{S}<\widehat{h}_{S}$, there exists two thresholds $\widehat{h}_{L} \in(0,1)$ and $\bar{z}_{m} \in\left(z_{l}, 1\right)$ such that:

(i) Three-layer offshoring is an equilibrium if and only if $h_{L} / h_{S}<\widehat{h}_{L}$ and $z_{m}>\bar{z}_{m}$.

(ii) Otherwise, offshoring is independent of $z_{m}$. If $z_{h} h_{I}>\widehat{h}_{L}$ there is no offshoring in equilibrium, while if $z_{h} h_{I}<\widehat{h}_{L}$ two-layer offshoring is an equilibrium.

Relative to Proposition 1, the main new result is the effect of the domestic communication cost $h_{S}$. Consistently with the results in Section IV, if $h_{S}$ is sufficiently low, then three-layer offshoring may cease to emerge in equilibrium, and the condition that determines the emergence of offshoring is independent of $z_{m}$. In particular, notice that whenever $h_{S} \rightarrow h_{L}$, as in Section IV, the condition $h_{L} / h_{S}<\widehat{h}_{L}$ cannot possibly hold (because $\widehat{h}_{L}<1$ ), which explains why we did not observe three-layer teams emerging in equilibrium in that case.

Interestingly, straightforward differentiation also indicates that the positive effect of $z_{m}$ on the left-hand-side of equation (11) is increasing in the level of $h_{S} \cdot{ }^{18}$ This implies that the positive effect of $z_{m}$ on the attractiveness of an equilibrium with three-layer offshoring is not only discreetly higher in the case with inefficient southern communication costs, but it is also the case that this marginal effect of $z_{m}$ smoothly increases as communication costs in the South become worse. ${ }^{19}$

Moreover, because the choice between no offshoring and two-layer offshoring is independent of $z_{m}$ and $h_{S}$, we can conclude that:

${ }^{18}$ This follows from

$$
\frac{\partial^{2}\left(\frac{z_{h}-z_{m} \frac{h_{L}}{h_{S}}-z_{l}\left(1-\frac{h_{L}}{h_{S}}\right)}{h_{I}\left(1-z_{m}\right)}\right)}{\partial z_{m} \partial h_{S}}=\frac{h_{L}\left(1-z_{l}\right)}{h_{S}^{2} h_{I}\left(1-z_{m}\right)^{2}}>0 .
$$

${ }^{19}$ As in Section III, we can also show that the possibility of forming three-layer teams is necessary for 
Corollary 2 If $h_{S}<\widehat{h}_{S}$ in equilibrium offshoring is (weakly) increasing in the skill level $z_{m}$ of middle-skilled agents. Furthermore, the positive effect of $z_{m}$ is (weakly) increasing in domestic communication costs $h_{S}$ in the South.

To sum up, this section has generalized the results in Section III and IV to the case in which local communication costs in the South are high, but not prohibitive. Consistently with the results above, we have found that middle skills can play a crucial role in bringing out the emergence of offshoring, but only when communication technologies in the South are sufficiently inefficient.

\section{Overall Effect of Middle Skills}

So far, we have divided the analysis in two regions: $h_{S} \geq \widehat{h}_{S}$ (Section III) and $h_{S}<\widehat{h}_{S}$ (Sections IV and V). Because the threshold $\widehat{h}_{S}$ depends itself on $z_{m}$, one may worry that by increasing $z_{m}$ we may jump from one region to another discontinuously. This is not the case. In particular, when we substitute the expression for $\widehat{h}_{S}$ in the left-hand side of (11) (the profits from three layer offshoring when $h_{S}<\widehat{h}_{S}$ ), we obtain exactly the left-hand side of condition (5) (the profits from three layer offshoring when $h_{S} \geq \widehat{h}_{S}$ ). In other words, the profits from three-layer offshoring are continuous in $z_{m}$ when we cross $\widehat{h}_{S}$. Since the right-hand side of these conditions is identical in both cases, the positive effect of $z_{m}$ on three-layer offshoring (or simply offshoring) holds globally. In sum,

Corollary 3 In equilibrium, offshoring is (weakly) increasing in the skill level $z_{m}$ of middle-skilled agents. Furthermore, the positive effect of $z_{m}$ is (weakly) increasing in domestic communication costs $h_{S}$ in the South.

We have assumed throughout the paper that $s_{l}$ is high relative to $s_{m}$ and $s_{h}$, which ensures that some low-skilled agents are self-employed in equilibrium. The case in which some middle-skilled agents are self-employed in equilibrium delivers very similar results. In particular, the model continues to predict that the partial effect of $z_{m}$ on offshoring is (weakly) increasing in $h_{S} \cdot{ }^{20}$ The main difference is that, consistently with the results at the end of Section IV, the effect of $z_{m}$ on offshoring may now be negative for sufficiently low $h_{S}$.

the emergence of offshoring whenever

$$
z_{h} h_{I}>\widehat{h}_{L}>\frac{h_{L}}{h_{S}}
$$

\footnotetext{
${ }^{20}$ In this case $w_{l}=\left[1-h_{S}\left(1-z_{l}\right)\right] z_{m}$ and $w_{m}=z_{m}$. This can be used to show that in the case of three layer offshoring or two layer offshoring with low types $\partial^{2} w_{h} / \partial z_{m} \partial h_{S}>0$, while in the case of two layer offshoring with medium types $\partial^{2} w_{h} / \partial z_{m} \partial h_{S}=0$.
} 


\section{EVIDENCE}

The simple model above was useful to understand the characteristics of target countries that lead to international offshoring. In this section we underscore the main empirical implication of the model and present evidence that suggests that it is supported by the data. In our theory, when southern communication costs are high, offshoring increases with the ability of medium-skilled agents (Corollary 3). The intuition is that, in some cases, in order for high-skilled agents in the North to benefit from offshoring, they need to add a layer of local managers that allows them to economize on international communication costs. In order for middle-skilled agents to serve this role, they need to be sufficiently skilled and their opportunity cost must be sufficiently low. Hence, the main implication of the model is that in countries where local communication technology is relatively bad (so middle managers can only lead small and inefficient southern teams), offshoring increases with the ability of middle-skilled managers. In contrast, in countries where communication technology is good, these middle managers will organize local teams and so more talented middle-skilled agents may not result in more offshoring, but only in more productive local teams.

The main empirical prediction of the theory can then be expressed as:

The volume of offshoring increases more with intermediate skills in countries where communication technology is relatively bad than in countries where communication technology is relatively good.

Note that this implication of our theory takes as given the level of international communication technology $h_{I}$, as well as the level of within multinational local communication technology $h_{L}$, and focuses on the level of local communication technology, $h_{S}$, as the source of cross-sectional variation in the data. To illustrate this, consider a northern firm that is deciding where to offshore, as in our theory. Then our empirical strategy assumes that this firm faces the same $h_{I}$ and $h_{L}$ in any host country, but can choose where to offshore depending on the host-country's $h_{S}$.

To contrast this prediction with the data, we use data from the World Bank's World Development Indicators (WDI). We use data on FDI inflows as a fraction of GDP as a measure of offshoring, ${ }^{21}$ the percentage of agents in the relevant age range enrolled in secondary school $(S S E)$ as a measure of intermediate skills, and an index of communication costs constructed using data on telephone, computer and internet usage. The Appendix includes a description of the factor analysis that leads to this index as well as more details

\footnotetext{
${ }^{21}$ We divide by GDP since the absolute level of offshoring in the model can be arbitrarily determined by choice of productivity and population size. We are aware that FDI is an imperfect measure of the volume of offshoring (see Lipsey, 2003). Unfortunately, however, data on the operations of offshoring facilities is not available for a large-cross section of host countries.
} 
on the raw series. Using the index we divide countries into two sets. Countries with bad communication technology $(B C I=1$, for bad communication index $)$ and countries with good communication technology $(B C I=0)$. The Appendix lists both sets of countries. We use a dummy variable for communication costs instead of the continuous index. ${ }^{22}$ This is because offshoring is independent of $h_{S}$ whenever $h_{S} \geq \widehat{h}_{S}$, so the model does not predict a strictly monotonic effect of communication technology. Throughout the analysis we use averages of these variables for the decade 1993-2002. We use the set of 122 countries for which we have complete data for all the variables of interest.

The raw data is presented in Figure 1. The figure also presents the corresponding regression lines, using a quantile regression with medians. All results presented in this section use quantile regressions to diminish the influence of outliers. The Appendix includes a discussion of quantile regressions and all results using OLS. In the graph, and in all OLS results in the Appendix, we eliminate two countries from the sample: Luxembourg and Equatorial Guinea. Both of these countries have extraordinarily high FDI over GDP ratios. This is probably the result of the predominance of some industries and their small size, characteristics that our model is not designed to address. All quantile regressions do include these two observations. Figure 1 shows that at first glance the prediction of the theory does well. The regression line for countries with bad communication has a larger slope than for countries with good communication. In countries with good communication technology the slope is in fact negative, which is consistent with the prediction of the theory described at the end of Sections IV and V.

The coefficients of both regression lines are presented in the first two columns of Table 1 (standard errors in parenthesis. $* * *, * *, *$ denote significant coefficients at $1 \%, 5 \%$ and $10 \%$ levels). One may think that the result is due to the fact that the countries with good communication all have SSE values higher than 70\%, while the larger sample of countries with low communication have values of SSE throughout the $[0 \%, 100 \%]$ range. This does not seem to be the case. If we restrict the sample of countries with poor communication to the ones that have $S S E>70$ (which results in a set of 31 countries), we obtain similar qualitative results. The coefficient on SSE then becomes .1665 (.0646) which is significant at a $5 \%$ level. Hence, for the rest of the empirical study we use the whole sample of countries.

${ }^{22}$ The cutoff used to build the dummy variable $B C I$ is 0.5 , which corresponds to one-half standard deviation above the mean communication index. All our results are robust to increasing or decreasing this cutoff by one-quarter standard deviation. Consistent with the model, if we lower the threshold even more (say to the mean communication index, that is, 0 ) the results become insignificant although they have the predicted sign. The reason is that we are mixing countries for which the effect of middle skills should be positive with countries for which the effect should be negative (such as Kuwait), which leads to insignificant coefficients. 
Figure 1: FDI and Secondary Education

(1993-2002 Averages)

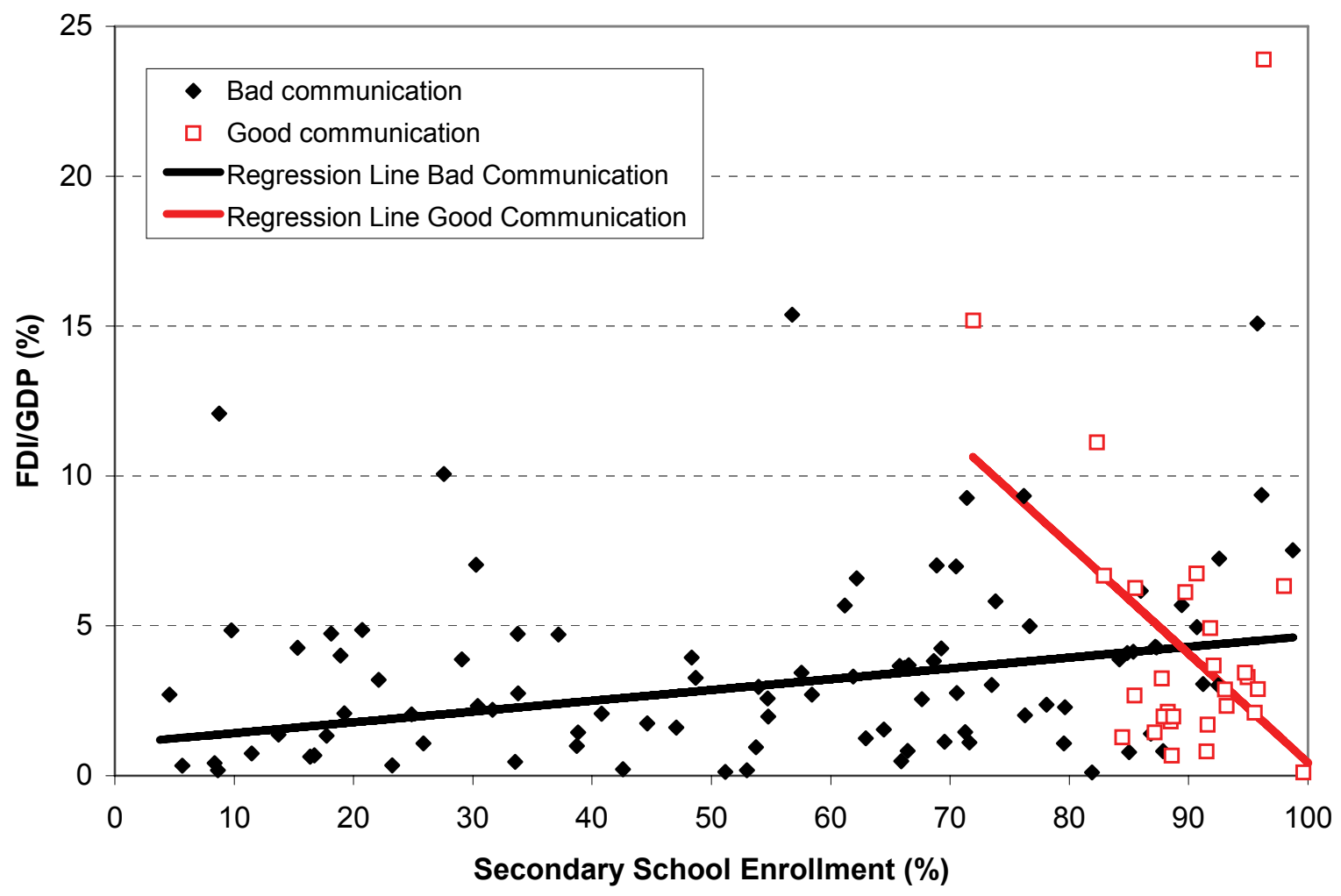

Source: World Bank Development Indicators. All data points are averages for the decade 1993-2002. For visibility we left out Luxemburg and Equatorial Guinea with FDI/GDP equal to 459.47 and 43.84 respectively. The regression line coefficients and estimation procedure are reported in the text.

We next seek to estimate more efficiently the interaction effect between the BCI dummy and secondary school enrollment by running a specification that incorporates the whole set of countries and includes an interaction term, together with the BCI dummy and the level of SSE in the regression. The third column of Table 1 presents these results. The prediction of our theory is that the interaction term should be positive and significant, and this is what we find in Table 1. This result does not depend on the particular construction of our index, as the coefficient on the interaction term is positive and significant at the $5 \%$ level using each of the components of the index separately.

A potential problem with these results is that secondary school enrollment may be highly correlated with other factors that, one may reasonable argue, influence the level of FDI/GDP, although they are not part of our theory. One of these factors is GDP per capita. In particular, our data on FDI inflows includes horizontal FDI, that is, FDI aimed at producing and selling products in these countries. Because GDP per capita is 
a proxy for market potential, it could have an independent effect on the ratio FDI/GDP. The fourth column of Table 1 presents the results if we add GDP per capita to our empirical specification. It is clear that this hardly changes our results. The coefficient of the interaction term remains positive and significant. The coefficient of GDP per capita is not significant at the $10 \%$ level.

Table 1: Median Regression Results

\begin{tabular}{|c|c|c|c|c|c|c|}
\hline \multicolumn{7}{|c|}{$\begin{array}{l}\text { Dependent Variable: } \frac{F D I}{G D P} \\
\text { Years: } 1993-2002 \text { Averages }\end{array}$} \\
\hline Constant & $\begin{array}{l}1.06^{*} \\
(.64)\end{array}$ & $\begin{array}{l}36.74^{* *} \\
(12.87)\end{array}$ & $\begin{array}{l}36.74^{* * *} \\
(8.72)\end{array}$ & $\begin{array}{l}36.74^{* * *} \\
(8.45)\end{array}$ & $\begin{array}{l}-3.35 \\
(12.43)\end{array}$ & $\begin{array}{l}19.87 \\
(11.15)\end{array}$ \\
\hline$S S E$ & $\begin{array}{l}.036 * * * \\
(.011)\end{array}$ & $\begin{array}{l}-.363^{* *} \\
(.143)\end{array}$ & $\begin{array}{l}-.363^{* * *} \\
(.0971)\end{array}$ & $\begin{array}{l}-.352^{* * *} \\
(.097)\end{array}$ & & $\begin{array}{l}-.379^{* * *} \\
(.085)\end{array}$ \\
\hline$S S E * B C I$ & & & $\begin{array}{l}.398^{* * *} \\
(.098)\end{array}$ & $\begin{array}{l}.390 * * * \\
(.097)\end{array}$ & & $\begin{array}{l}.413^{* * *} \\
(.086)\end{array}$ \\
\hline$B C I$ & & & $\begin{array}{l}-35.68^{* * *} \\
(8.75)\end{array}$ & $\begin{array}{l}-35.11^{* * *} \\
(8.76)\end{array}$ & $\begin{array}{l}4.01 \\
(12.26)\end{array}$ & $\begin{array}{l}-19.21 \\
(10.96)\end{array}$ \\
\hline$P S E * B C I$ & & & & & $\begin{array}{l}-.043 \\
(.126)\end{array}$ & $\begin{array}{l}-.183^{*} \\
(.108)\end{array}$ \\
\hline$P S E$ & & & & & $\begin{array}{l}.087 \\
(.123)\end{array}$ & $\begin{array}{l}.203 \\
(.105)\end{array}$ \\
\hline$\frac{G D P}{P o p}$ & & & & $\begin{array}{l}-.095 \\
(.371) \\
\end{array}$ & $\begin{array}{l}-.206 \\
(.329)\end{array}$ & $\begin{array}{l}-.177 \\
(.313) \\
\end{array}$ \\
\hline Countries & $B C I=1$ & $B C I=0$ & All & All & All & All \\
\hline \# of Obs. & 93 & 29 & 122 & 122 & 122 & 122 \\
\hline
\end{tabular}

Our theory makes a clear distinction between agents with different skill levels. These agents perform different roles in the economy and have different occupations in equilibrium. It is important, therefore, that these results are not just driven by some average level of education, but by secondary or intermediate levels of education. In particular, the prediction of our theory for the effect of the skill level of medium-skilled agents on 
FDI/GDP does not hold for low-skilled agents. Thus, to study whether the results presented reflect the forces in our theory, we repeat the regression presented in the fourth column of Table 1 but using instead primary school enrollment (PSE). The results are presented in the fifth column of Table 1. We find reassuring that the interaction term involving primary school enrollment and communication technology appears statistically insignificant. To emphasize this conclusion we also run the regression using both levels of schooling. In column 6 , the interaction term of secondary schooling remains positive and significant, while the one for primary schooling is insignificant. Overall, we interpret our results as strongly suggestive of the existence of a disproportionately positive effect of middle skills on offshoring in countries with bad communication technology.

In linking the main prediction of the theory with this empirical exercise in Table 1 we have equated secondary school enrollment to intermediate skills. In our setup, however, this intermediate skills are the highest skill level in developing countries. Therefore, a reasonable concern is that the actual empirical counterpart of our intermediate skills is probably some measure of tertiary education -which leaves post-graduate education as the counterpart of high skills in the North- or a combination of tertiary and secondary education. Of course, secondary education is a requirement for tertiary education and so the union of tertiary education and secondary educations is equivalent to using SSE. We repeated the exercise in Table 1 and obtain the exact same qualitative results using tertiary education. Given that many managers in less developed countries are agents without college we prefer to call intermediate skills the union of secondary and tertiary school enrollment and so, to save on space, we do not present the results using only tertiary education.

A concern with the results above is that our index of local communication costs is constructed using data on telephone, computer, and internet usage, which may lead to endogeneity of our communication cost index. FDI can determine how much agents use these technologies, which would lead to biased coefficients. Following this logic, a natural conjecture is that FDI decreases the index of bad communication technology, and it does so more the higher secondary school enrollment. However, this mechanism would tend to bias the interaction term towards zero. Hence, this type of endogeneity would tend to reinforce our finding that the true coefficient is positive and significant. Of course, endogeneity may take other forms and so this argument does not definitely solve the endogeneity problem. We would need better data on the state of communication technology, not the use of technology, in order to rule out other potential sources of endogeneity.

Table 2 in the Appendix presents the same six regressions using OLS instead of quantile regressions. The results are qualitatively similar. We obtain all the right signs and all the relevant coefficients are significant, although only at the $10 \%$ level. Admittedly, this body of evidence, although consistent with the theory, is more suggestive than conclusive. 
Bilateral data (and preferably at the industry level) seems necessary to develop a much more complete taxonomy of the characteristics that make some countries good targets of offshoring. ${ }^{23}$ We leave this for future research.

\section{CONCLUSIONS}

The theory we develop in this paper makes two main points. First, that the ability of organizations to change their organizational form and make use of agents with different talents is important to understand the decision of organizations to offshore part of their production. In our theory this organizational change takes the form of an extra intermediate layer of management, and so the ability of these managers becomes a crucial determinant of the extensive margin of offshoring. Second, that the local communication technology of a country determines the opportunity costs of workers - since it determines the characteristics of local teams- and therefore the desirability of such a country as a target for offshoring. In order to understand this second argument, and the interaction between both of them, it is necessary to have a general equilibrium theory where these opportunity costs and the offshoring decisions are both determined in equilibrium.

These two main arguments lead to several empirical implications from which we have highlighted one that can be readily contrasted with the data. Namely, that the ability of middle-skilled agents increases offshoring by relatively more in countries where communication technology is bad, than in countries where communication technology is relatively good. The empirical results we present are very encouraging in that they show that this is in fact the case in the data, and that this relationship is not driven by GDP per capita, or primary schooling. The model has other predictions that we have not studied empirically: most importantly, that offshoring will happen in larger teams (and with more layers) in countries where low-skilled agents are readily available. Since this large teams

are also more efficient (output per worker is higher) this also provides an implication for the productivity of the firms that offshore to these countries. An empirical investigation of this prediction requires, of course, data on firm characteristics and so we leave it for future work.

\footnotetext{
${ }^{23}$ See Ramondo (2005) for an attempt along these lines.
} 


\section{REFERENCES}

Aitken, B. and A.Harrison (1999), "Do Domestic Firms Benefit from Foreign Direct Investment? Evidence from Panel Data," American Economic Review, 89(3), pp. 605-618.

Antràs, Pol (2003), "Firms, Contracts, and Trade Structure," Quarterly Journal of Economics, 118:4, pp. 1375-1418.

Antràs, Pol, Luis Garicano and Esteban Rossi-Hansberg (2006), "Offshoring in a Knowledge Economy," Quarterly Journal of Economics, forthcoming.

Feenstra, Robert C. (1998), "Integration of Trade and Disintegration of Production in the Global Economy," Journal of Economic Perspectives, 12:4, pp. 31-50.

Friedman, Thomas L. (2005), The World Is Flat: A Brief History Of The Twenty-first Century, Farrar, Straus and Giroux.

Garicano, Luis (2000), "Hierarchies and the Organization of Knowledge in Production." Journal of Political Economy, 108(5), pp. 874-904.

Garicano, Luis and Esteban Rossi-Hansberg (2005), "Organization and Inequality in a Knowledge Economy." Mimeo, Princeton University.

Garicano, Luis and Esteban Rossi-Hansberg (2006), "The Knowledge Economy at the Turn of the Twentieth Century: The Emergence of Hierarchies." forthcoming in the Journal of the European Economic Association P\&P.

Helpman, Elhanan (1984), "A Simple Theory of International Trade with Multinational Corporations", Journal of Political Economy, 92:3, pp. 451-471.

Larraín, Felipe, Luis F. López-Calva, and Andrés Rodríguez-Clare (2001), "Intel: A Case Study of Foreign Direct Investment in Central America," Chapter 6 in ed. Felipe Larraín, Economic Development in Central America, vol. I: Growth and Internationalization. Harvard University Press.

Lipsey, Robert E. (2003), "Foreign Direct Investment and the Operations of Multinational Firms: Concepts, History, and Data," in E. Kwan Choi and James Harrigan (eds.), Handbook of International Trade, Blackwell: Oxford, UK.

Ramondo, Natalia (2005), "Size, Geography, and Multinational Production," mimeo University of Chicago.

Spar, Debora (1998), "Attracting High Technology Investment: Intel's Costa Rican Plant," FIAS Occasional Paper 11. Washington D.C.: World Bank.

Yeaple, Stephen (2003), "The Role of Skill Endowments in the Structure of U.S. Outward FDI," Review of Economics and Statistics, August, 85(3), pp. 726-734. 


\section{APPENDIX: DATA AND EMPIRICAL ANALYSIS}

\section{Communication Index}

The raw data used to construct our index of the state of communication technology in each country is taken from the online version of the World Bank's World Development Indicators (WDI). The index is constructed using data on telephone, computer and internet usage. The three series are, respectively, (i) fixed line and mobile phone subscribers per 1,000 inhabitants; (ii) personal computers per 1,000 inhabitants; and (iii) internet users per 1,000 inhabitants. To build the communication index we first average the three indicators for the 1993-2002 period, and then we perform a factor analysis of the correlation matrix. We used the first factor as the basis for the country-by-country communication index, which has mean 0 and standard deviation equal to 1 .

A 0.5 cutoff in this index yields a division of countries into 93 countries with "bad" communication technologies $(B C I=1)$ and 29 countries with "good" communication technologies $(B C I=0)$. We list the countries in each group below.

Countries with low communication costs $(B C I=0)$ :

Australia, Austria, Belgium, Canada, Cyprus, Germany, Denmark, Estonia, Finland, France, Hong Kong, Iceland, Ireland, Israel, Italy, Japan, Korea, Luxembourg, Malta, Netherlands, New Zealand, Norway, Portugal, Slovenia, Spain, Sweden, Switzerland, United Kingdom, United States.

Countries with high communications costs $(B C I=1)$ :

Albania, Algeria, Armenia, Bahrain, Bangladesh, Barbados, Belize, Benin, Bolivia, Botswana, Brazil, Bulgaria, Burkina Faso, Burundi, Cambodia, Cape Verde, Chad, Chile, Colombia, Costa Rica, Cote d'Ivoire, Croatia, Czech Republic, Djibouti, Dominica, Ecuador, Egypt, El Salvador, Equatorial Guinea, Eritrea, Etiopia, Fiji, Gambia, Georgia, Ghana, Greece, Grenada, Guatemala, Guinea, Guyana, Hungary, Indonesia, Jamaica, Jordan, Kenya, Kuwait, Lao, Latvia, Lithuania, Madagascar, Malaysia, Maldives, Mauritania, Mauritius, Mexico, Moldova, Mongolia, Morocco, Mozambique, Nicaragua, Niger, Nigeria, Oman, Panama, Papua New Guinea, Paraguay, Peru, Philippines, Poland, Romania, Samoa, Saudi Arabia, Seychelles, Slovakia, South Africa, St. Kitts and Nevis, St. Lucia, St. Vincent and the Grenadines, Swaziland, Syria, Tanzania, Togo, Tonga, Trinidad and Tobago, Tunisia, Ukraine, Uruguay, Vanuatu, Venezuela, Vietnam, Yemen, Zambia, Zimbabwe.

\section{Empirical Analysis}

The empirical analysis in Table 1, (Section VI) presents a quantile (median) regression, or least absolute value model; the model chooses by maximum likelihood the vector of regressors $b$ to minimize $\sum_{i}|y-x b|$ (rather than, as in OLS, $\left.\sum_{i}(y-x b)^{2}\right)$. Such an estimator is preferred whenever there are substantial outliers in the dependent variable, which are given excessive weight in the calculation of the regression by OLS. 
Our results remain however unchanged if we proceed by OLS and restrict our attention to a sample of countries with $F D I / G D P<40 \%$. Such restriction excludes from the analysis 2 extreme outliers: Luxembourg, with an average FDI/GDP for the sample period of $459.5 \%$ and Equatorial Guinea with an average of $43.84 \%$. These should be compared to a sample distribution with quantiles $1.44 \%, 2.93 \%$, and $4.86 \%$. For completeness, in Table 2 below we present OLS results in exactly the same order as in Table 1 in the body of the paper.

Table 2: OLS Regression Results

\begin{tabular}{|c|c|c|c|c|c|c|}
\hline \multicolumn{7}{|c|}{$\begin{array}{l}\text { Dependent Variable: } \frac{F D I}{G D P} \\
\text { Years: 1993-2002 Averages }\end{array}$} \\
\hline \multirow{2}{*}{ Constant } & $2.08^{* * *}$ & 21.91 & $21.91^{* *}$ & $22.86^{* *}$ & -10.15 & 1.65 \\
\hline & $(.70)$ & $(15.19)$ & (10.79) & $(11.39)$ & $(19.41)$ & (19.81) \\
\hline \multirow{2}{*}{$S S E$} & $.026 * *$ & -.192 & -.193 & -.190 & & $-.240^{*}$ \\
\hline & $(.011)$ & $(.168)$ & $(.120)$ & $(.120)$ & & $(.126)$ \\
\hline \multirow{2}{*}{$S S E * B C I$} & & & $.219^{*}$ & $.221 *$ & & $.261 * *$ \\
\hline & & & $(.120)$ & $(.121)$ & & $(.127)$ \\
\hline \multirow{2}{*}{$B C I$} & & & $-19.83^{*}$ & $-20.14^{*}$ & 10.31 & -.035 \\
\hline & & & $(10.82)$ & $(10.93)$ & $(19.13)$ & $(19.65)$ \\
\hline \multirow{2}{*}{$P S E * B C I$} & & & & & -.110 & -.244 \\
\hline & & & & & $(.196)$ & $(.203)$ \\
\hline \multirow{2}{*}{$P S E$} & & & & & .151 & .268 \\
\hline & & & & & $(.194)$ & $(.201)$ \\
\hline \multirow{2}{*}{$\frac{G D P}{P o p}$} & & & & -.119 & -.0001 & -.168 \\
\hline & & & & $(.441)$ & $(.378)$ & $(.445)$ \\
\hline Countries & $B C I=1$ & $B C I=0$ & All & All & All & All \\
\hline \# of Observations & 92 & 28 & 120 & 120 & 120 & 120 \\
\hline$R^{2}$ & 0.04 & 0.05 & 0.07 & 0.07 & 0.05 & 0.08 \\
\hline
\end{tabular}

